\title{
Natural Product Analysis over the Last Decades
}

Authors

Affiliation

\section{Andrew Marston, Kurt Hostettmann}

Laboratory of Pharmacognosy and Phytochemistry, School of Pharmaceutical Sciences, University of Geneva, Geneva, Switzerland

\author{
Key words \\ - analysis \\ - separation \\ - chromatography \\ - spectroscopy \\ - hyphenated techniques
}

received Oct. 21, 2008

revised January 2, 2009

accepted January 13, 2009

Bibliography

DOI $10.1055 / \mathrm{s}-0029-1185379$

Published online March 4, 2009

Planta Med 2009; 75: 672-682

(c) Georg Thieme Verlag KG

Stuttgart · New York .

ISSN 0032-0943

Correspondence

Prof. Kurt Hostettmann

Laboratory of Pharmacognosy

and Phytochemistry

School of Pharmaceutical

Sciences

University of Geneva

Quai Ernest-Ansermet 30

1211 Geneva 4

Switzerland

Phone: +41223793401

Fax: +41223793399

kurt.hostettmann@unige.ch

\section{Abstract \\ $\nabla$}

Progress in natural product chemistry has always been strongly linked to innovations in analytical technology. The characterisation of metabolites in complex mixtures requires sophisticated techniques, which should provide good sensitivity and selectivity as well as structural information on the constituents of interest. This review out-

\section{Introduction}

$\nabla$

Ever since antiquity, Man has strived to be able to study and analyse the products of the natural world around him. Up to the twentieth century, when plants were the source of most therapeuticals, observation of characteristic structural elements of plants under the microscope was the main method used for their quality control, while chemical analysis of constituents played a minor role. As the importance of the contribution of the individual natural products to the pharmacological and biological activities of the organism was realised, so did the demand increase for more suitable qualitative and quantitative analytical methods. At first, adequate methods did not exist for the analysis of the very complex mixtures provided, for example, by plant extracts. It was not until the 1950s that the breakthrough in the analysis of natural products was provided by the application of chromatographic separation methods and spectroscopic identification and structure elucidation techniques. These have been progressively improved over the last few decades, with the result that very efficient chemical, physical, biological and, most importantly, physicochemical, methods are now available [1]. The other innovation has been the progressive reduction in sample size necessary for analysis.

The general term "separation techniques" refers to various forms of chromatography and also elec- lines the most important chromatographic and spectral techniques which have been introduced in the field of natural products. Although there has been a very rapid evolution of methods over the last 50 years, the introduction of highthroughput screening programmes require even more efficient and sensitive methodologies which yield adequate on-line information for metabolite structure determination.

\section{Chromatography \\ $\nabla$}

\section{History}

Chromatography has developed from a rudimentary tool, introduced at the beginning of the 20th century for the separation of pigments into an array of techniques capable of dealing with the most complex analytical and purification problems of natural products [3]. The developments can basically be divided into three important landmarks: the first introduction of chromatography; the contribution played by Martin in the 1950s; and the introduction of commercially- 


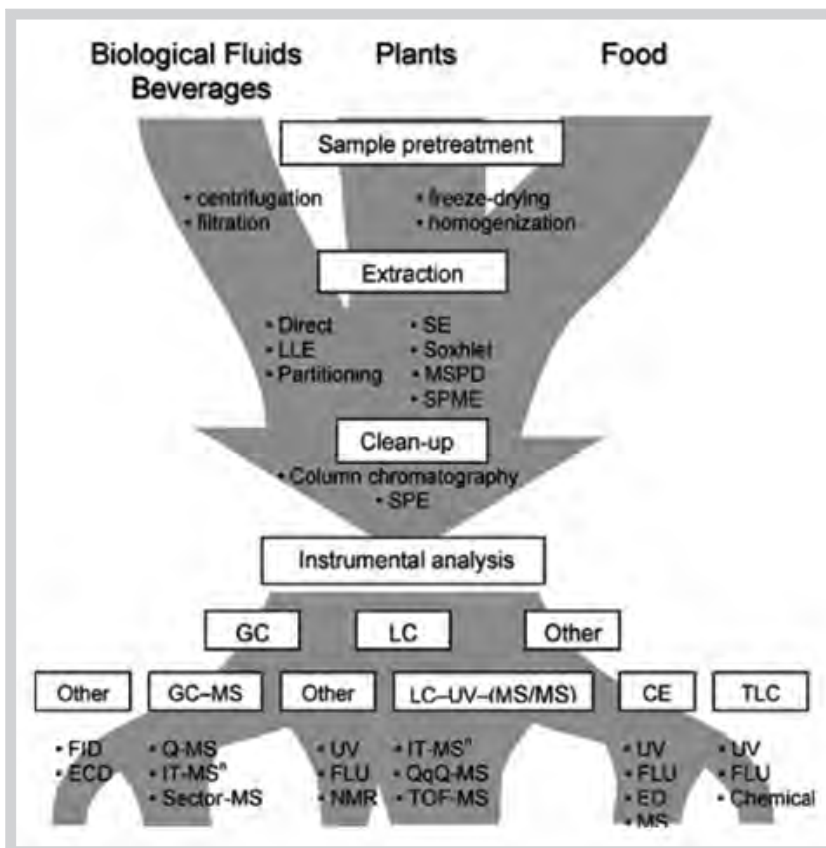

Fig. 1 Strategies for the analysis of flavonoids in biological fluids, beverages, plants and food. Abbreviations: LLE, liquid-liquid extraction; SE, solvent extraction; MSPD, matrix solid-phase extraction; SPME, solid-phase micro-extraction; SPE, solid-phase extraction; GC, gas chromatography; LC, liquid chromatography; MS, mass spectrometry; MS/MS, tandem mass spectrometry; CE, capillary electrophoresis; TLC, thin layer chromatography; FID, flame ionisation detection; ECD, electron capture detection; Q, quadrupole; QqQ, triple-quadrupole; IT, ion-trap, FLU, fluorescence; NMR, nuclear magnetic resonance; TOF, time-of-flight and ED, electrochemical detection (reprinted with permission from ref. [1]).

available high-performance liquid chromatography (HPLC) equipment in the 1970 s.

Tswett introduced column adsorption chromatography at the beginning of the 20th century, initially for the separation of plant pigments. The first printed description of the method came with a lecture in 1903 at the Warsaw Society of Natural Sciences entitled "On a new category of adsorption phenomena and their application to biochemical analysis". This was the starting point of liquid chromatography and, in a paper published in 1906, Tswett took the subject further with the notion of the "chromatogram" and its development by using different eluents [4]. By the end of the 1930s, column adsorption chromatography (nowadays known as normal phase chromatography) had become a widely used separation technique for plant extracts and natural products

Although the method provided access to numerous compounds, resolution was low and difficulties were experienced with watersoluble samples. It was not until the work of Martin and coworkers that further significant advances were made in the chromatography of natural products, with the invention of partition chromatography (liquid-liquid chromatography, LLC) [5]. Martin described partition chromatography, in which a liquid stationary phase is immobilised on a solid support, as a marriage between Tswett's adsorption-based chromatography and countercurrent solvent extraction. In the 1941 paper, gas-liquid chromatography (GLC) is also anticipated: "the mobile phase need not to be a liquid but may be a vapour. Very refined separations of volatile substances should therefore be possible in a column in which a permanent gas is made to flow over a gel impregnated with a non-volatile solvent". The development of partition chromatography earned Martin and Synge the 1952 Nobel Prize in chemistry.

Martin and coworkers also introduced paper chromatography using filter paper sheets impregnated with water or other liquids; this became the first chromatographic microanalytical technique [6]. However, the slow migration rates of paper chromatography have resulted in this technique being overtaken by thin-layer chromatography (TLC), in which the application of thin layers of adsorbent on glass plates originated in Russia [7].

Finally, GLC was introduced in 1952 [8] and became widely available in the 1960s. It is suitable for small, volatile compounds. Sensitive universal detection was provided by flame ionisation (FID). GLC (or GC) is ideal for the analysis of complex mixtures such as those found in essential oils. In one run, it is possible to separate hundreds of constituents and identify them by comparison with a data base. Quantitation is performed using the FID or by GC-MS, although many operators prefer FID data to total ion current (TIC) response data, since MS response factors for different analytes often vary. An example is given in $\bullet$ Fig. $\mathbf{2}$, in which a Japanese mugwort essential oil of the leaves was analysed by GC and 192 components were identified.

The general term "chromatography" has evolved to include an amazing array of techniques and variants of these techniques. Some of the most frequently employed chromatographic methods are given in $\bullet$ Table 1.

\author{
Chromatographic method \\ Liquid-solid chromatography \\ Paper \\ Gas liquid chromatography (GLC) \\ Thin-layer chromatography (TLC) \\ High-performance liquid chromatography (HPLC) \\ Ultra-performance liquid chromatography (UPLC) \\ Supercritical fluid chromatography (SFC) \\ Liquid-liquid chromatography (LLC) \\ Countercurrent chromatography (CCC) \\ lon-exchange chromatography (IEC) \\ Capillary electrophoresis (CE) \\ Ion-pair chromatography \\ Hydrophobic interaction chromatography (HIC, HILIC) \\ Size exclusion chromatography (SEC) \\ Affinity chromatography
}

Separation mechanism
adsorption
(adsorption), partition
adsorption, partition
adsorption, partition
adsorption, partition
adsorption, partition
adsorption, partition
partition
partition
ion exchange
charge
ion pair formation, ion interaction
(adsorption), partition
size of analyte
biological affinity

Table 1 The major chromatographic methods. 


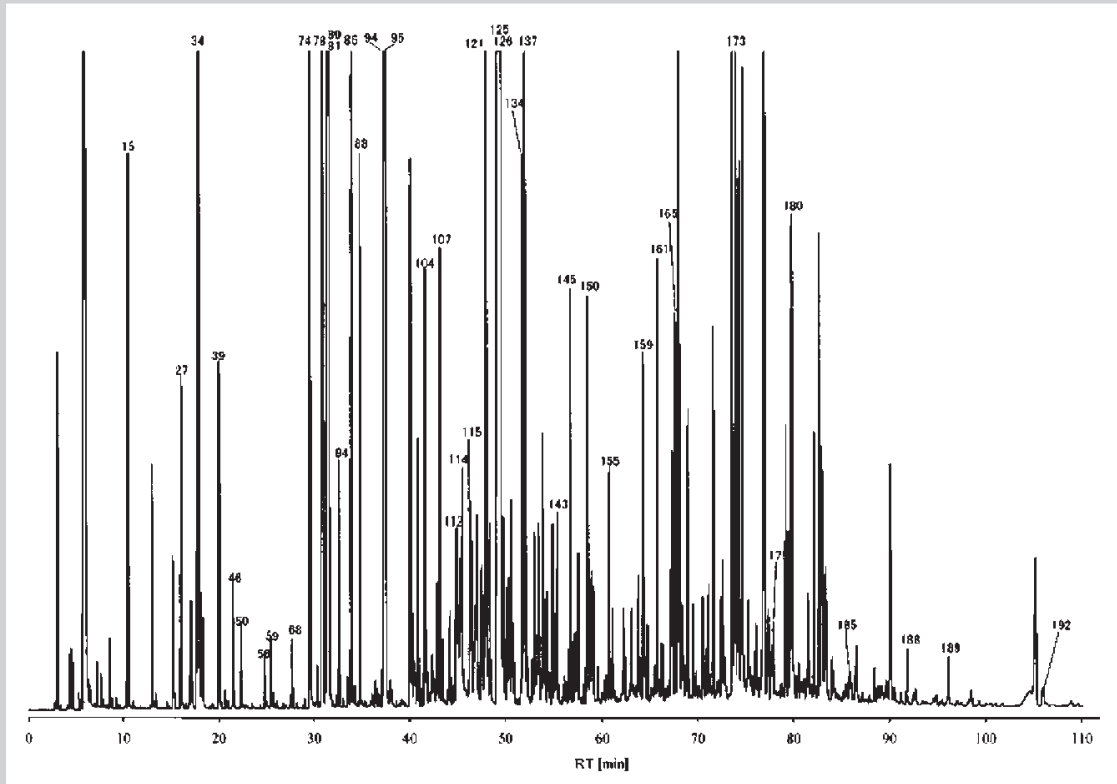

Fig. 2 Gas chromatogram of an extract from the leaves of Japanese mugwort (Artemisia princeps, Asteraceae) obtained by steam distillation under reduced pressure. Column: HP 6890 equipped with DB-Wax bonded-phase fused silica capillary column and a flame ionisation detector (FID) (Reprinted with permission from ref. [9]).

\section{Planar chromatographic techniques}

TLC: Thin layer chromatography (TLC) is the only chromatographic method offering the option of presenting the result as an image. Furthermore, TLC is the sole technique in which all the components of the sample are included in the chromatogram. In contrast, HPLC and GC are selective and not all of the compounds in the sample are included in the display.

The real breakthrough in TLC came through the work of Stahl, who introduced the use of calcium sulphate as binder, and standardised layer thickness and chromatographic development [10]. Not only does the technique give visual results but it excels in its simplicity and is low in cost. Parallel analysis of samples is possible, sample capacity is high and results are obtained rapidly. TLC is flexible and multiple detection is possible. It is an ideal screening method in biological and chemical analysis, providing identification and qualitative results, determination of adulteration, together with quantitative and semiquantitative determination. In conjunction with microorganisms and other biological agents, TLC bioautography can be used to screen for bioactivities (see below) [11]. The disadvantages of TLC are a lack of automation, the problems of reproducibility which sometimes occur and the lack of accuracy in quantitation. Nevertheless, TLC will remain a fast and simple micro-technique of chromatography [12].

In HPTLC, the plates are precoated with a stationary phase with a typical mean particle size of $5 \mu \mathrm{m}$. The plates give better separations and reproducibility than normal precoated TLC plates (mean particle size $12 \mu \mathrm{m}$ ) and they also allow more sensitive detection. Shorter developing distances are required. The number of theoretical plates is in the 5000 range [13], while for HPLC the range is $6-10000$. The separation power of HPTLC is still lower than that of HPLC and the latter is preferred for quantitative determination. Merck also offers HPTLC plates with spherical particles, which gives faster chromatography and better separation power. A water-resistant layer is available from Merck and for RP-18 plates, $100 \%$ water can be used with these plates.

For herbal extracts, regulatory agencies often recommend fingerprint chromatography for proper identification purposes. HPTLC is ideal in this instance and excellent examples can be found in the literature $[13,14]$. HPTLC is also ideally suited for the preliminary screening of plant extracts before HPLC analysis.

A recent review gives an in-depth summary of TLC and HPTLC [15] and the subject is amply treated elsewhere in this Special Issue.

\section{Other planar chromatographic techniques}

None of the other planar techniques have had the impact of TLC. In overpressured layer chromatography (OPLC), a horizontal thin-layer chromatography plate is covered by an elastic cushion. Pressure is applied to the cushion so that separations are performed on the TLC plate in the absence of a vapour phase. Sample development is rapid and high efficiencies can be achieved [16]. Automated multiple development (AMD) allows a gradient-driven separation in TLC and can produce remarkable separations of complex mixtures [17].

\section{Column chromatographic techniques}

High performance liquid chromatography (HPLC): The most remarkable advances in chromatography have occurred in the domain of HPLC, despite the fact that the technique itself has only been in existence for about 40 years. The year 1967 was a landmark in the introduction of HPLC ( $\bullet$ Fig. 3 ), with papers from Horvath, Huber and Scott (see, for example, [18]), but the first automatic liquid chromatograph with gradient elution was an amino acid analyser described by Moore and coworkers in 1958 [19]. Until the advent of HPLC, most natural product separations were performed by open-column, paper or thin-layer chromatography. Open-column chromatography was time-consuming and tedious, often requiring a large amount of sample. With paper chromatography and TLC, very small samples could be analysed and the resolution and reproducibility improved. However, quantitation was still inadequate and resolution of similar compounds difficult. Gas chromatography provided excellent resolution but the restriction to volatile samples (less than $20 \%$ of organic compounds can be separated by gas chromatography) meant that derivatisation was often necessary. A technique was needed which could separate water-soluble, thermally-labile, nonvolatile compounds with speed, precision and high resolution. HPLC fulfilled 


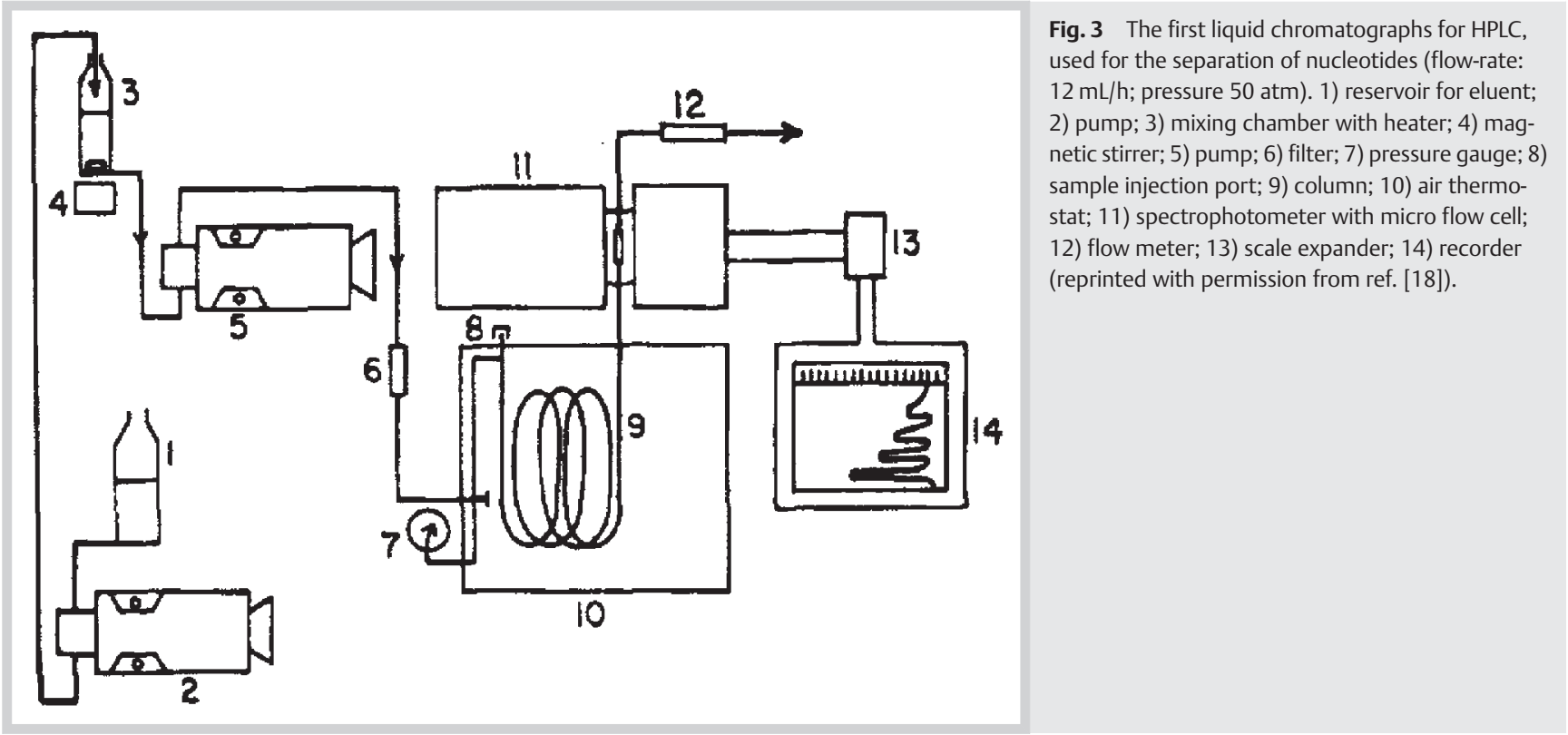

these criteria and is now one of the most powerful tools in analytical chemistry, with the ability to separate, identify and quantitate the compounds present in any sample that can be dissolved in a liquid. The viscosity of liquids is higher than that of gases by a factor of 100 - hence the need for pressure in the columns and the original name "high-pressure liquid chromatography". But "pressure" was replaced by "performance" as particles got smaller and columns became shorter.

The wide variety of stationary and mobile phases should give a large potential for finding suitable separation conditions. However, at first only relatively large particles were available. The introduction of small porous silica particles with a diameter of approximately $10 \mu \mathrm{m}$ radically changed the situation, as did the production of chemically-bonded phases, notably the reversedphase (RP) octadecyl (RP-18) and octyl (RP-8) materials. Standardisation of silica surfaces by defined hydroxylation, application of very pure silica, improvement in bonding and end-capping procedures now give very versatile reversed-phase systems, with high selectivity, high separation power, robustness, high stability and efficiency. Reviews on equipment and instrumentation are published periodically in journals such as Analytical Chemistry - for example, by LaCourse [20]. An excellent early comprehensive review on the analysis of natural products was provided by Kingston in 1979 [21]. Since then, there have been numerous developments but the same basic conditions predominate, i.e., octadecylsilyl columns and methanol-water or acetonitrile-water as eluent.

High-performance liquid chromatography has become by far the most widely used chromatographic technique. In fact, the segments liquid chromatography, mass spectrometry and thermal analysis account for US $\$ 4.4$ billion of the global US $\$ 20$ billion analytical instrumentation market.

Analytical HPLC: Applications of HPLC are found in hundreds of areas. One of the most important of these is the area of quality control in the pharmaceutical industry.

In the last decade, high-performance liquid chromatography (HPLC) has been one of the most frequently used techniques for the separation of natural products in complex biological matrices such as crude plant extracts. For chemotaxonomic purposes, the botanical relationships between different species can be shown by chromatographic comparison of their chemical composition. Chromatograms, which are used as fingerprints, are compared with authentic samples and unknown substances to permit identification of drugs and/or search for adulteration. HPLC is thus the best suited technique for an efficient separation of the crude extracts, as shown by Sakakibara et al. [22] who claim to have found a method capable of quantifying every polyphenol in vegetables, fruits and teas. For this purpose they used a Capcell pak C18 UG120 $(250 \times 4.6 \mathrm{~mm}, \mathrm{~S}-5,5 \mu \mathrm{m})$ column and gradient elution with sodium phosphate (pH3.3) and 10\% methanol, and 70\% methanol. The method allowed the determination of aglycones separately from glycosides. Information could also be obtained about simple polyphenols in the presence of more complex polycyclic polyphenols. Quantitative determination was achieved for a total of 63 different food samples.

High-performance liquid chromatography (HPLC) is used routinely in phytochemistry to "pilot" the preparative isolation of natural products (optimisation of the experimental conditions, checking of the different fractions throughout the separation) and to control the final purity of the isolated compounds.

The level of structural information that can be obtained has been, however, limited by the available LC detectors. Detectors based on UV, fluorescence, refractive index, light scattering or electrochemistry provide good detection and sensitivity but without the possibility of detailed structural information. The introduction of hyphenated techniques such as LC/UV with photodiode array detection (LC/UV-DAD) and LC coupled with mass spectrometry (LC/MS) has provided real advances in on-line metabolite structure determination [23]. These coupled techniques are discussed below.

Ultra-high-pressure liquid chromatography (UHPLC): The introduction of spherical particles has led to a reduction in the size of the phase particles to $3 \mu \mathrm{m}$ in 1978 and $1.5 \mu \mathrm{m}$ in 1990. This means that column efficiency is improved and shorter columns are possible, leading to shorter separation times [24]. Column lengths of 10 to $25 \mathrm{~cm}$ ( 8000 to 10000 plates $/ \mathrm{m}$ ) have now dropped to around $6 \mathrm{~cm}$ for $3 \mu \mathrm{m}$ particles in order to attain the same plates $/ \mathrm{m}$. The analysis time correspondingly drops by a fac- 


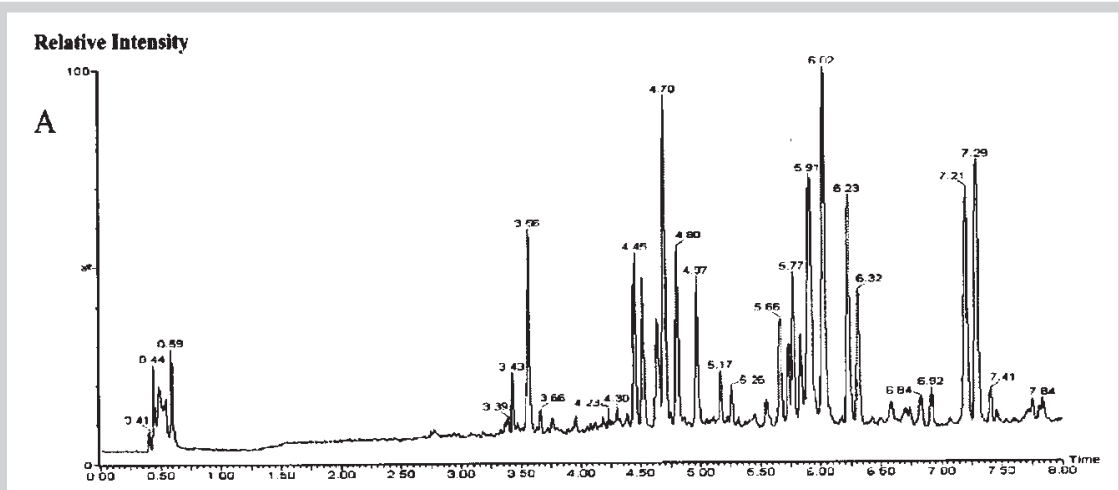

Fig. 4 A UPLC/TOFMS electrospray total ion current chromatogram (TIC) and B HPLC/UV chromatogram $(203 \mathrm{~nm})$ of steamed Panax notoginseng (Araliaceae) (reprinted with permission from ref. [25]).

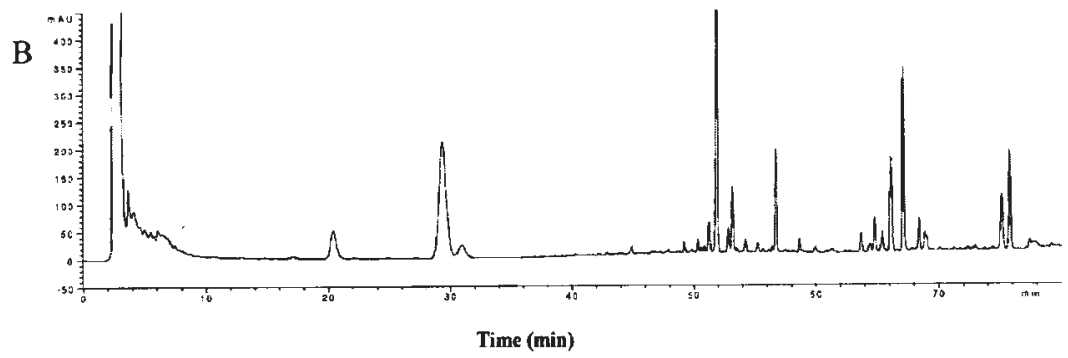

tor of around 4. At the same time, obviously there is a corresponding increase in the pressure necessary to perform the separation, giving rise to the term "ultra-high-pressure liquid chromatography (UHPLC)". The trend is now towards columns with $1 \mu \mathrm{m}$ particles, which would give pressures of around 7000 bar. In ultra-performance liquid chromatography (UPLC), the trade mark of Waters Acquity systems, introduced in 2004, particle sizes of around $1.7 \mu \mathrm{m}$ are used, at a pressure of $15000 \mathrm{psi}$ (1000 bar). Applications in the separation of natural products are beginning to appear, as in the investigation of the constituents of raw and steamed ginseng root, Panax notoginseng, Araliaceae. UPLC was performed on a Waters Acquity system with a $100 \times 2.1 \mathrm{~mm} \mathrm{C}_{18} 1.7 \mu \mathrm{m}$ column and a mobile phase gradient consisting of (A) $0.1 \%$ formic acid in water and (B) acetonitrile containing $0.1 \%$ formic acid. The UPLC system was connected to an orthogonal acceleration TOF mass spectrometer and was compared to a classical LC/UV analysis. Identification of peaks was achieved by comparison with an in-house database containing 96 protopanaxadiol-type ginsenosides. The ultra-fast chromatography displayed higher reproducibility when compared with traditional HPLC ( $\odot$ Fig. 4) [25].

Capillary electrophoresis (CE): Capillary electrophoresis is an analytical technique which provides high separation efficiency and short run times. Several modes of CE are available: (1) capillary zone electrophoresis (CZE), (2) micellar electrokinetic chromatography (MEKC), (3) capillary gel electrophoresis (CGE), (4) capillary isoelectric focusing, (5) capillary isotachophoresis, (6) capillary electrochromatography (CEC) and (7) nonaqueous CE. The simplest and most versatile CE mode is CZE, in which the separation is based on differences in the charge-to-mass ratio and analytes migrate into discrete zones at different velocities. Anions and cations are separated in CZE by electrophoretic migration and electro-osmotic flow (EOF), while neutral species co-elute with the EOF. Applications of CE for the analysis of natural products have been well documented [26-28]. CE is especially suitable for the separation of flavonoids as they are negatively charged at higher pH values [28].
Thin-layer chromatography, HPLC, GC and capillary electrophoresis (CE) are all useful methods for fingerprinting and analysing plant drugs. While HPLC has high precision, sensitivity and reproducibility, it requires lengthy pretreatment of samples to remove residual solid material and eliminate irreversible adsorptive losses on the solid support matrix. Capillary electrophoresis is becoming increasingly recognised as a fast and efficient analytical separation technique but the reproducibility and selectivity are inferior to HPLC.

Countercurrent chromatography: The technique of countercurrent chromatography (CCC) basically involves the separation of a sample between two non-miscible liquid phases. One of these phases is kept stationary in a coiled column or a chamber by a centrifugal force, while the other phase (mobile phase) is pumped from one extremity of the column to the other. As a sample is passed through the system, it partitions between the two phases. Solute retention depends only on the partition coefficient: adsorption is precluded by the absence of a solid phase. Instruments with rotating coiled columns are often constructed in such a way that they produce a planetary motion [29], accompanied by a hydrodynamic equilibrium between the two solvent phases. This aids the partition of the sample between the two phases. With CCC, the stationary phase occupies $50-80 \%$ of the total volume, whereas in HPLC, this figure is around 5\%. This implies that the capacity of CCC is higher. Another advantage over HPLC is that fewer theoretical plates are necessary in CCC to achieve a given resolution. Although HPLC is generally considered to be faster than CCC, this is offset by the time required for HPLC column clean-up and the rapid deterioration of the HPLC columns, which necessitates expensive replacement.

Countercurrent chromatography is another advance of the 20th century. It is basically a development of countercurrent distribution (CCD), a method developed in the 1940s and 1950s for the batchwise (Craig distribution) or continuous (O'Keefe distribution) fractionation of mixtures. Modern countercurrent chromatography finds its origins in pioneering work by Y. Ito at the NIH in the USA [30]. 
Efficiencies reported in analytical-scale CCC clearly indicate that this method will not compete with the ubiquitous technique of analytical HPLC but it has some important uses in methods development, in micro-scale separations and in the screening for new bioactive compounds in crude extracts. Analytical HSCCC is also an attractive method for interfacing with mass spectrometers because of the reduced flow-rates.

\section{Spectroscopic Techniques}

$\nabla$

Ultraviolet (UV)

Structure elucidation by ultraviolet spectroscopy is of great importance because this is the most sensitive of all spectroscopic techniques. And since the introduction of derivative spectroscopy, the sensitivity has improved even further, so that UV spectroscopy is perfect for trace analysis.

\section{Infrared (IR)}

Fourrier-transform infrared spectroscopy (FT-IR) has a far greater sensitivity than classical IR and allows far more rapid measurements. With complete scans available in 0.1 to 1 second, it has been possible to couple FT-IR with GC (even capillary columns). In combination with spectral databanks, the method is very powerful for the identification of unknowns in mixtures.

\section{Mass spectrometry (MS)}

In this field, developments have been exceptionally numerous. Starting with electron impact (EI) spectra, a whole series of ionisation techniques has been developed, starting with chemical ionisation $(\mathrm{CI})$ and passing through field desorption (FD), fastatom bombardment (FAB) and thermospray (TSP), to the atmospheric-pressure ionisation (API) techniques electrospray ionisation (ESI) and atmospheric pressure chemical ionisation (APCI). In the matrix-assisted laser desorption/ionisation (MALDI) technique, sample ions are usually analysed by a time-of-flight (TOF) mass analyser. MALDI-TOF-MS has advantages over other methods, including high speed of analysis, good sensitivity (with an ability to determine mass with an accuracy better than 1 part per million) and good tolerance towards contaminants.

\section{Nuclear magnetic resonance (NMR)}

The most powerful method for the structural analysis of natural products comes from the observation of nuclear magnetic resonance (NMR) in 1945 by Felix Bloch at Stanford University [31] and Edward Purcell at Harvard University [32]. Bloch and Purcell received the first Nobel Prize in NMR. Initial manipulation of the nuclear spin carried out by Hahn was essential for further development of experiments such as "insensitive nuclei enhanced by polarisation transfer" (INEPT) [33], which is the basis of many modern pulse sequence experiments. Ernst and colleagues in the 1970s had the idea of acquiring a two-dimensional (2D) spectrum by applying two separate radiofrequency pulses with different increments between the pulses [34]. Ernst was subsequently awarded the Nobel Prize in 1991 for his work. A whole series of 2D correlation experiments, such as COSY, HMBC, HSQC are now widely employed in structure elucidation by NMR.

Quantitative NMR ( $q N M R$ ) has a long history, dating almost from the discovery of NMR. qHNMR has enormous potential in the identification, characterisation and discovery of bioactive natural products [35] and in the area of metabolome analysis. It is treated in another part of this Special Issue.
Capillary NMR (CapNMR): New probe technologies have increased NMR sensitivity. With a capillary probe $(5 \mu \mathrm{L}$ total volume and $1.5 \mu \mathrm{L}$ active volume) and an RF coil wound into a solenoid configuration, shimming is easier and minute amounts of deuterated solvents are consumed. With samples of $10 \mu \mathrm{g}$, 1D proton spectra can be acquired in 5 minutes and 2D gradient COSY NMR spectra within 1.5 hours. HSQC and HMQC experiments are acquired on $30 \mu \mathrm{g}$ samples within 5 hours and HMBC experiments with $70 \mu \mathrm{g}$ in 10 to 15 hours [36].

\section{Hyphenated Techniques}

$\nabla$

Hyphenated techniques are defined as methods combining two or more analytical techniques (usually a separation and a spectroscopic technique) into one integrated technique [37]. The first to be used was HPLC coupled with UV spectroscopy (LC-UV) and GC was combined with MS, to be followed later by HPLC coupled with MS. However, GC is restricted to volatile constituents and the ionisation techniques used in LC-MS generally only give information about the molecular ion and the basic fragments. NMR alone, with the array of experiments it includes, goes the furthest towards determination of structures and, thus with HPLC, is a very powerful combination.

\section{LC-UV}

A computer-controlled photodiode array (PDA) detector for liquid chromatography was reported in 1976 [38] but it took several years until the first commercial photodiode array detector became available in the early 1980 s [39] (O Fig. 5). This allowed the running of a chromatographic separation with simultaneous detection at different wavelengths. The potential of PDA in the field of natural product analysis was rapidly recognised. Notable early examples include the identification of phenolic compounds by the concomitant use of on-line UV-vis shift reagents enhancing the spectral information content [40]. However, the structural information provided by electronic spectra is quite limited.

\section{LC-MS}

HPLC coupled with mass spectrometry is the analytical technique which has had the most impact of all the hyphenated methods. Even laboratory personnel without a spectroscopic background are now making everyday use of LC-MS instrumentation.

The idea of interfacing mass spectrometry with liquid chromatography had been around ever since the successful implementation of GC-MS. Due to inherent incompatibilities of a liquid mobile phase and a high vacuum mass analyser, LC-MS was initially exploited by very few groups. In LC-MS, there are three general problems: the amount of column effluent that has to be introduced in the MS vacuum system, the composition of the eluent and the type of compounds to be analysed. Many interfaces have been developed in order to cope with these factors [41]. The interfaces must accomplish nebulisation and vaporisation of the liquid, ionisation of the sample, removal of excess solvent vapour and extraction of the ions into the mass analyser. To date, no real universal interface has been constructed; each interface has characteristics that are strongly dependent on the nature of the compounds for which they are used.

However, with the introduction of atmospheric pressure ionisation (API), ions could be generated outside the high vacuum part of the mass spectrometer. The atmospheric pressure chemical ionisation (APCI) interface was first reported in 1983 [42], soon 


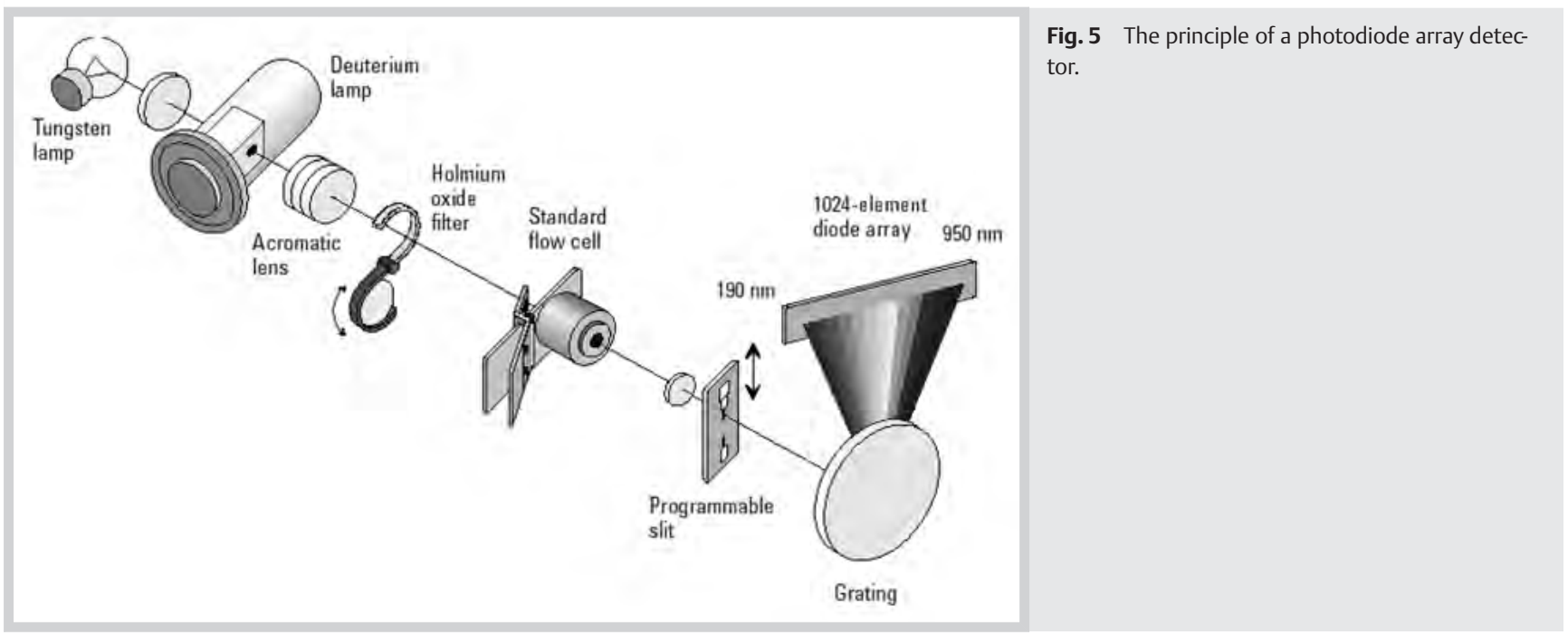

followed by the electrospray interface in 1985 [43]. The potential of API-MS for the dereplication of natural products has been extensively studied [44]. Today, LC-MS is a powerful routine tool in numerous natural products laboratories around the world.

\section{LC-NMR}

Nuclear magnetic resonance (NMR) spectroscopy is the most powerful and versatile technique for the structure elucidation of natural products. The idea of using NMR as an LC detection device was put forward at the end of the 1970s [45]. The inherently low sensitivity of NMR and the state of probe design hampered its practical implementation. A first generation of sensitive NMR flow-probes was developed in the early 1990s [46]. First applications of LC-NMR to natural products analysis were soon to follow [47], and the potential of the concerted use of on-line spectroscopy involving LC-NMR was subsequently described [48]. It is a breakthrough in the sense that a comprehensive NMR structural analysis of individual compounds in an extract has become feasible on the HPLC scale. In conjunction with other on-line detectors, structure elucidation with just the effluents of an analytical HPLC separation has become a reality.

The technique of LC-NMR is well illustrated by the structure determination of two isomeric meroterpenoid naphthoquinones from Cordia linnaei (Boraginaceae). These were in a fraction obtained from the dichloromethane extract of the roots but could not be separated by semi-preparative HPLC. On-flow LC- ${ }^{1} \mathrm{H}-\mathrm{NMR}$ (๑ Fig.6), combined with a TOCSY selective pulse experiment and a COSY experiment, allowed attribution of the signals at C21 to $\mathrm{C}-24$ in the side chain and hence differentiation of the two isomers [49].

Small-volume (30-120 $\mu \mathrm{L})$ flow-probes offer a mass sensitivity (signal-to-noise ratio per mass unit) that is much greater than that of traditional NMR probes. Using a $600 \mathrm{MHz}$ magnet, the limit of ${ }^{1} \mathrm{H}$ detection of a compound with MW 500 is roughly $100 \mathrm{ng}[50]$.

Continuous-flow LC-NMR: Continuous-flow (or on-flow) experiments were the first LC-NMR experiments to be introduced. Their disadvantage is the limited residence time of the analyte in the NMR flow-cell and consequently the detectable amount of analyte is restricted to about $10 \mu \mathrm{g}$ [50].

Stopped-flow LC-NMR: In this variant of LC-NMR, the analyte of interest (observed in the HPLC chromatogram) is kept in the flow-cell by stopping eluent pumping. The longer residence time in the flow-cell allows longer acquisition times and consequently 2D NMR spectra can be run [51].

\section{LC-SPE-NMR}

In LC-SPE-NMR, chromatographic peaks eluted from a reversedphase HPLC column are passed one by one through small solidphase extraction columns (SPE cartridges) in order to remove the analyte from the HPLC mobile phase. The SPE cartridges are subsequently dried with nitrogen gas and the analytes desorbed with a deuterated solvent for NMR spectroscopy [52].

LC-SPE-NMR is the latest step in a long series of developments in LC-based on-line spectroscopy. Predecessors employing off-line LC-NMR were to be found in the late 1980s [53] but these early approaches were of limited use due to the low sensitivity of the NMR probes at this time. The concept was revived with the advent of newer NMR flow cells [54,55], and now cryogenicallycooled flow probes and peak collection with a sophisticated SPE interface are used [50].

Another alternative is to use the off-line combination of HPLC-SPE with CapNMR for the analysis of complex mixtures such as natural product extracts, as reported for the rapid identification of sesquiterpene lactones and esterified phenylpropanoids from Thapsia garganica (Apiaceae) fruits [56].

\section{HPLC-CD}

Natural products frequently contain stereocentres which can be studied by chiroptical methods such as circular dichroism (CD). Chiroptical stereoanalysis of individual compounds in an extract has become feasible with the introduction of an LC-CD detector [57].

\section{Other hyphenated techniques [51]}

These include SFC-NMR, chiral LC-NMR, ion-exchange chromatography-NMR, CCC-NMR, gel permeation chromatographyNMR. 


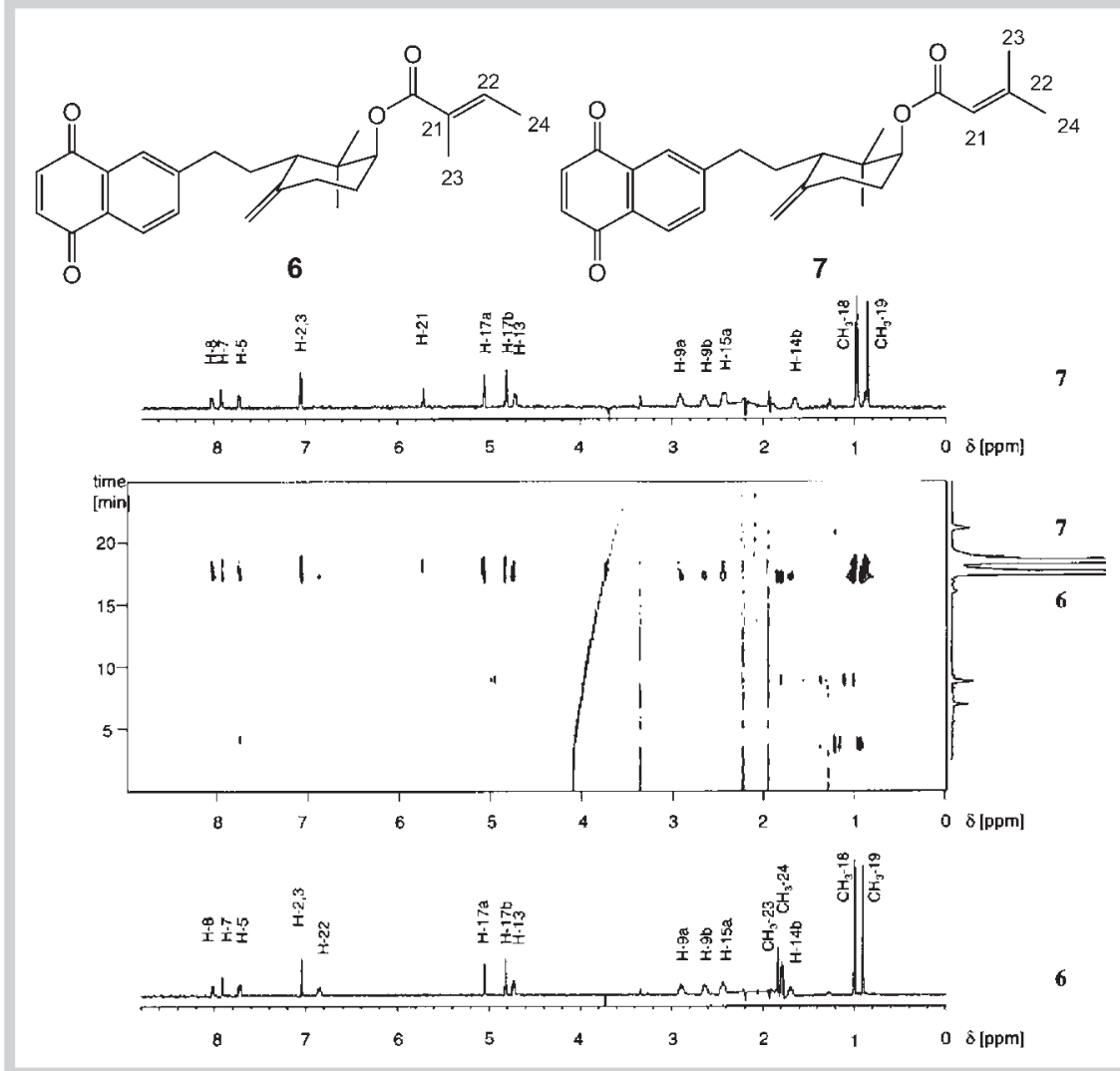

Fig. 6 Two-dimensional LC- ${ }^{-} \mathrm{H}-\mathrm{NM}$ R chromatogram of a fraction from the dichloromethane extract of the roots of Cordia linnaei (Boraginaceae), including the on-flow ${ }^{1} \mathrm{H}$-NMR spectra of the naphthoquinones $\mathbf{6}$ and $\mathbf{7}$ in acetonitrile-water (reprinted with permission from ref. [49]).

\section{Dereplication}

$\nabla$

In the search for bioactive constituents from plants or from other natural sources, the approach adopted often consists only of bioactivity-guided fractionation of crude extracts [58]. By following this procedure alone, there is a risk of unnecessarily isolating known natural products. Furthermore, potential lead compounds which are not active in the given bioassays will simply be missed. The rediscovery of known compounds poses a significant challenge in natural product research and calls for ingenious dereplication strategies, i.e., ways of avoiding the time-consuming isolation of known constituents. This can be achieved by the application of hyphenated techniques such as LC-MS or LC-UV and LC-NMR at the earliest stage of separation [59]. These analyses are valuable to detect compounds with interesting structural features and to target their isolation.

\section{Bioanalysis \\ $\nabla$}

Despite the fact that a large number of the drugs in clinical use are of natural origin, little is known about their mechanism of action and their pharmacokinetic properties. Suitable bioanalytical methods for the detection of natural products in biological matrices, for their structural characterisation and the quantitative analysis of their metabolites are needed. Of the techniques described in this review, LC-MS is one of the most powerful for bioanalysis. An excellent review has been published which looks at all aspects of LC coupled to tandem MS for the determination of bioactive natural products and their metabolites in biological fluids: their extraction, their analysis, optimisation of MS conditions and applications of LC-MS [60].

\section{Bioassay as an Analytical Tool \\ $\nabla$}

No review of analytical methods for natural products should be without a mention of biological or pharmacological tests. These biological screening methods are an essential complement to the chemical screening methods mentioned above. The domain of biological analysis is vast and just one or two aspects will be mentioned here.

In order to obtain results fast and with the minimum of outlay in terms of expense and material, simple so-called "benchtop bioassays" can be used. Some of these are listed in 0 Table 2.

A certain number of the simple bioassays involve the use of thinlayer chromatography since this is an inexpensive, reproducible and simple means of rapidly screening plant extracts and fractions $[11,61,62]$. TLC has several attractive features: parallel sample processing for high sample throughput; accessibility of the samples for post-chromatographic evaluation free of time constraints; minimal sample clean-up [62]. Bioautography combines

Table 2 Some simple benchtop bioassays.

Antifungal (Cladosporium cucumerinum, Candida albicans)

Antibacterial (Escherichia coli, Staphylococcus aureus, Bacillus subtilis)

Molluscicide (Biomphalaria glabrata, Bulinus globosus)

Piscicide (Oryza latipes, Tilapia sp.)

Larvicide (Aedes aegypti, Anopheles sp., Culex sp.)

Spermicide (human spermatozoids)

Insecticide (Diabrotica sp., Aphis sp.)

Antifeedant (Spodoptera sp., Epilachna varivestis)

Antioxidant and free-radical scavengers

Germination inhibition 

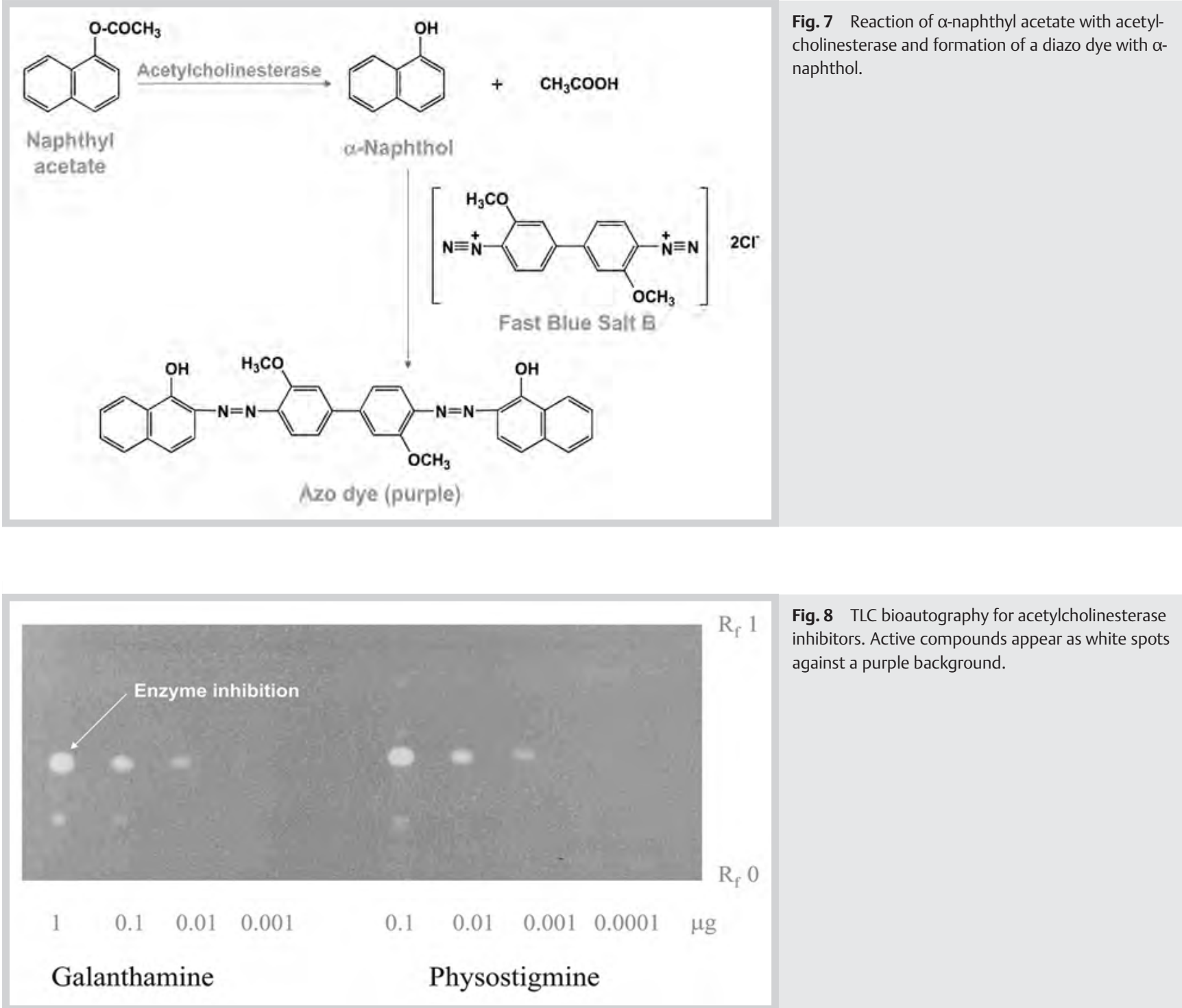

Fig. 8 TLC bioautography for acetylcholinesterase inhibitors. Active compounds appear as white spots against a purple background.

thin-layer chromatography with a bioassay in situ and allows localisation of active constituents in a complex matrix. It can be employed for the target-directed isolation of these constituents. Common methods of bioassay for thin-layer separations are based on the inhibition of growth or activity of a test organism, such as mould spores, yeast cells, bacteria, cell organelles or enzymes.

One of the first TLC-based tests was developed by Homans and Fuchs in 1970 [63]. It is applicable to microorganisms that can grow directly on the TLC plate and uses the spore-producing fungus Cladosporium cucumerinum. Growth of the fungus is seen as a grey colouration on the plate, while inhibition zones are white. A TLC bioautographic assay has been introduced to screen plant extracts and other samples for inhibition of acetylcholinesterase activity and to aid in the search for new potential anti-Alzheimer drugs. The test relies on the cleavage reaction of acetylcholinesterase on 1-naphthyl acetate, to form 1-naphthol, which in turn reacts with Fast Blue B Salt to give a purple-coloured diazonium dye ( Fig. 7) [64], except in regions containing acetylcholinesterase inhibitors which show up as white spots. The plant-derived alkaloids galanthamine and physostigmine are used as reference compounds ( $\bullet$ Fig. 8).
For a rapid screening of plant extracts, coupling HPLC with a bioassay is possible. An example is the coupling of HPLC with the DPPH screening for radical scavengers [65]. The sample is separated over an HPLC column and the separated components detected by UV. At the same time, a part of the eluent is split off and reacted with a DPPH solution. Radical scavengers are observed as negative peaks at $517 \mathrm{~nm}$ ( Fig. 9). It is thus possible to correlate a peak in the chromatogram with an activity and to quantitate the activity.

Hyphenation of bioassays with HPLC-SPE-NMR is another exciting possibility [66].

\section{Outlook}

$\nabla$

What does the future have in store for the analysis of natural products? The tendency will be to follow analytical methods for environmental and biological problems, where the emphasis is principally on the search for smaller and smaller quantities and the use of more sensitive detection methods.

The trend is towards higher information content, faster analyses (one manufacturer of chromatography systems now advertises 


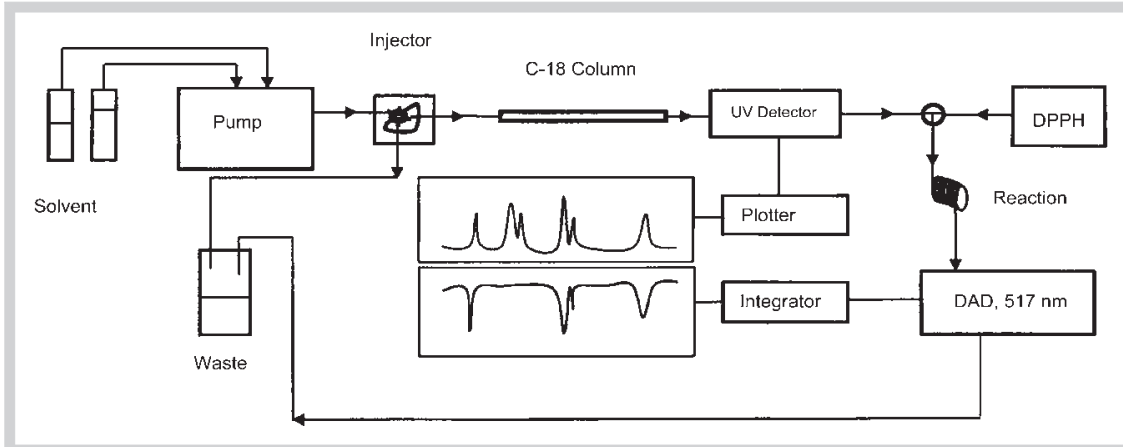

Fig. 9 HPLC coupled to an on-line assay with DPPH for radical scavengers (reprinted with permission from ref. [65]).

the ability to analyse 10 peaks in 10 seconds!), improved mass accuracy and separations of more complex mixtures using a combination of techniques. Increasing use will be made of UHPLC to for faster separation times and capillary NMR to accommodate smaller sample quantities.

In LC-MS, Q-TOFs and Trap-TOFs are presently very popular because they maximise the advantages of different mass analysers while minimising the disadvantages. Novel fragmentation technologies, such electron capture dissociation (ECD), electron transfer dissociation (ETD), and infrared multiphoton dissociation (IRMPD) are becoming progressively more accessible. Both nanoscale and multidimensional liquid chromatography technologies are working their way into stable commercial platforms. In proteomics, there is a movement away from the peptide-centred approach towards intact protein analysis. Sensitivity and resolution will always be areas that can be improved on for the analysis of complex peptide mixtures. In fact, "omics" applications, such as proteomics, glycomics, lipidomics and cellomics are pushing the boundaries of what can be done with different technologies - in particular LC-MS. And LC-MS is becoming important in the areas of fermentation monitoring, toxicology and the distribution of biologically active compounds in the environment (ecotoxicology). Single quadrupole mass spectrometry systems will most probably be a generic detector for an LC or UHPLC inlet. On the downside, very little has been done to reduce the losses between the atmospheric pressure area and the first vacuum stage of an LC-MS instrument. The speed and quantity of data analysis continues to be insufficient.

Finally, the ever-improving chromatographic tools combined with spectral techniques will make possible not only the investigation of the rapidly disappearing flora around us but will also allow study of precious items such as herbarium collections, unique specimens and microscopic organisms. The analysis of minute amounts of toxins will be within range of the scientist, giving a greater surveillance of the environment around him.

\section{Acknowledgments}

$\nabla$

The Swiss National Science Foundation (grant no. 2000063670.00 to K. Hostettmann) is gratefully acknowledged for financial support.

\section{References}

1 De Rijke E, Out P, Niessen WMA, Ariese F, Gooijer C, Brinkman UAT. Analytical separation and detection methods for flavonoids. J Chromatogr A 2006; 1112: 31-63

2 Schreier P, Herderich M, Humpf HU, Schwab W. Natural product analysis. Berlin: Springer; 2000

3 Marston A. Role of advances in chromatographic techniques in phytochemistry. Phytochemistry 2007; 68: 2786-2798

4 Tswett MS. Adsorptionsanalyse und chromatographische Methode. Anwendung auf die Chemie des Chlorophylls. Ber Dtsch Bot Ges 1906; 24 : 384-393

5 Martin AJP, Synge RLM. A new form of chromatogram employing two liquid phases. Biochem J 1941; 35: 1358-1368

6 Consden R, Gordon AH, Martin AJP. Qualitative analysis of proteins: a partition chromatographic method with paper. Biochem J 1944; 38: 224-232

7 Izmailov NA, Shraiber MS. Displacement chromatography. Farmatsia (Russ.) 1938; 3: 1-12

8 Martin AJP, James AT. Gas-liquid partition chromatography: the separation and micro-estimation of volatile fatty acids from formic acid to dodecanoic acid. Biochem J 1952; 50: 679-690

9 Umano K, Hagi Y, Nakahara K, Shoji A, Shibamoto T. Volatile chemicals identified in extracts from leaves of Japanese mugwort (Artemisia princeps Pamp.). J Agric Food Chem 2000; 48: 3463-3469

10 Stahl E. Thin-layer chromatography. II. Standardization, detection, documentation and application. Chemiker-Zeitung 1958; 82: 323-329

11 Hostettmann K, Terreaux C, Marston A, Potterat $O$. The role of planar chromatography in the rapid screening and isolation of bioactive compounds from medicinal plants. J Planar Chromatogr 1997; 10: 251-257

12 Stahl E. Dünnschicht-Chromatographie, ein Laboratoriumshandbuch, 2nd edition. Berlin: Springer; 1967

13 Reich E, Schibli. A. High-performance thin-layer chromatography for the analysis of medicinal plants. New York: Thieme Medical Publishers; 2007

14 Wagner H, Bladt S. Plant drug analysis: a thin-layer chromatography atlas, 2nd edition. Berlin: Springer; 1995

15 Sherma J. Planar chromatography. Anal Chem 2008; 80: 4253-4267

16 Tyihak E, Mincsovics E, Kalasz H. New planar liquid chromatographic technique - overpressured thin-layer chromatography. J Chromatogr 1979; 174: 75-81

17 Burger K. DC-PMD, Dünnschicht-Chromatographie mit GradientenElution im Vergleich zur Säulenflüssigskeits-Chromatographie. Z Anal Chem 1984; 318: 228-233

18 Horvath CG, Preiss BA, Lipsky SR. Fast liquid chromatography. Investigation of operating parameters and the separation of nucleotides on pellicular ion exchangers. Anal Chem 1967; 39: 1422-1428

19 Spackman DH, Stein WH, Moore S. Automatic recording apparatus for use in chromatography of amino acids. Anal Chem 1958; 30: 11901206

20 LaCourse WR. Column liquid chromatography: equipment and instrumentation. Anal Chem 2002; 74: 2813-2832

21 Kingston DGI. High performance liquid chromatography of natural products. J Nat Prod 1979; 42: 237-260

22 Sakakibara H, Honda Y, Nakagawa S, Ashida H, Kanazawa K. Simultaneous determination of all polyphenols in vegetables, fruits and teas. J Agric Food Chem 2003; 51: 571-581 
23 Lindon JC, Nicholson JK, Sidelmann UG, Wilson ID. Directly coupled HPLC-NMR and its application to drug metabolism. Drug Metab Rev 1997; 29: 705-746

24 Nguyen DT, Guillarme D, Rudaz S, Veuthey JL. Fast analysis in liquid chromatography using small particle size and high pressure. J Sep Sci 2006; 29: 1836-1848

25 Chan ECY, Yap SL, Lau AJ, Leow PC, Toh DF, Koh HL. Ultra-performance liquid chromatography/time of flight mass spectrometry based metabolomics of raw and steamed Panax notoginseng. Rapid Commun Mass Spectrom 2007; 21: 519-528

26 Issaq HJ. Capillary electrophoresis of natural products - I. Electrophoresis 1997; 18: 2438-2452

27 Issaq HJ. Capillary electrophoresis of natural products - II. Electrophoresis 1999; 20: 3190-3202

28 Tomas-Barberan FA. Capillary electrophoresis: a new technique in the analysis of plant secondary metabolites. Phytochem Anal 1995; 6: 177-193

29 Ito, Y. Golden rules and pitfalls in selecting optimum conditions for high-speed countercurrent chromatography. J Chromatogr A 2005; 1065: 145-168

30 Ito $Y$, Weinstein MA, Aoki I, Harada R, Kimura E, Nunogaki K. The coil planet centrifuge. Nature 1966; 212: 985-987

31 Bloch F, Hansen WW, Packard M. Nuclear induction. Physics Rev 1946; 69: 127

32 Purcell EM, Torrey HC, Pound RV. Resonance absorption by nuclear magnetic moments in a solid. Physics Rev 1946; 69: 37-38

33 Morris GA, Freeman $R$. Enhancement of nuclear magnetic resonance signals by polarization transfer. J Am Chem Soc 1979; 101: 760-762

34 Aue WP, Bartholdi E, Ernst RR. Two-dimensional spectroscopy. Application to nuclear magnetic resonance. J Chem Phys 1976; 64: 2229-2246

35 Pauli GF, Jaki BU, Lankin DC. Quantitative ${ }^{1} \mathrm{H}$ NMR: development and potential of a method for natural products analysis. J Nat Prod 2005; 68: $133-149$

36 Hu JF, Garo E, Yoo HD, Cremin PA, Zeng L, Goering MG, O'Neil-Johnson M, Eldridge GR. Application of capillary-scale NMR for the structure determination of phytochemicals. Phytochem Anal 2005; 16: 127-133

37 Jaroszewski JW. Hyphenated NMR methods in natural products research, part 1: Direct hyphenation. Planta Med 2005; 71: 691-700

38 Dessy RE, Reynolds WR, Nunn WG, Titus CA. New mini-computer automated linear photodiode arrays spectrometer system for high-resolution liquid chromatography. J Chromatogr 1976; 126: 347-368

39 George SA, Maute A. A photodiode array detection system: design, concept and implementation. Chromatographia 1982; 15: 419-425

40 Hostettmann K, Domon B, Schaufelberger D, Hostettmann M. On-line high-performance liquid chromatography - ultraviolet-visible spectroscopy of phenolic compounds in plant extracts using post-column derivatization. J Chromatogr 1984; 283: 137-147

41 Niessen WMA. State-of-the-art in liquid chromatography-mass spectrometry. J Chromatogr A 1999; 856: 179-197

42 Thomson BA, Danylewychmay L, Henion JD. LC-MS-MS with an atmospheric pressure chemical ionization source. Abstr Pap Am Chem Soc 1983: 19

43 Whitehouse CM, Dreyer RN, Yamashita M, Fenn JB. Electrospray interface for liquid chromatographs and mass spectrometers. Anal Chem 1985; 57: 675-679

44 Niessen WMA. Progress in liquid chromatography-mass spectrometry instrumentation and its impact on high-throughput screening. J Chromatogr A 2003; 1000: 413-436

45 Watanabe N, Niki E. Direct-coupling of FT-NMR to high-performance liquid-chromatography. Proc Jpn Acad Ser B 1978; 54: 194-199

46 Spraul M, Hofmann M, Dvortsak P, Nicolson JK, Wilson ID. High-performance liquid chromatography coupled to high-field proton nuclear magnetic resonance spectroscopy: application to the urinary metabolites of ibuprofen. Anal Chem 1993; 65: 327-330

47 Spring O, Buschmann H, Vogler B, Schilling E, Spraul M, Hoffmann M. Sesquiterpene lactone chemistry of Zaluzania grayana from on-line LC-NMR measurements. Phytochemistry 1995; 39: 609-612

48 Hostettmann K, Wolfender JL, Rodriguez S. Rapid detection and subsequent isolation of bioactive constituents of crude plant extracts. Planta Med 1997; 63: 2-10

49 Ioset JR, Wolfender JL, Marston A, Gupta MP, Hostettmann K. Identification of two isomeric meroterpenoid naphthoquinones from Cordia linnaei by liquid chromatography-mass spectrometry and liquid chromatography-nuclear magnetic resonance spectroscopy. Phytochem Anal 1999; 10: 137-142

50 Corcoran 0, Spraul M. LC-NMR-MS in drug discovery. Drug Discov Today 2003; 8: 624-631

51 Jaroszewski JW. Hyphenated NMR methods in natural products research, part 1: direct hyphenation. Planta Med 2005; 71: 691-700

52 Jaroszewski JW. Hyphenated NMR methods in natural products research, part 2: HPLC-SPE-NMR and other new trends in NMR hyphenation. Planta Med 2005; 71: 795-802

53 Wilcox $C D$, Phelan RM. The use of solid-phase extraction columns to effect simple off-line LC/MS, LC/NMR and LC/FTIR. J Chromatogr Sci 1986; 24: 130-133

54 Griffiths L, Horton R. Optimization of LC-NMR III - Increased signal-tonoise ratio through column trapping. Magn Reson Chem 1998; 36: 104-109

55 Nyberg NT, Baumann H, Kenne L. Application of solid-phase extraction coupled to an NMR flow-probe in the analysis of HPLC fractions. Magn Reson Chem 2001; 39: 236-240

56 Lambert M, Wolfender JL, Staerk D, Brogger Christensen S, Hostettmann $K$, Jaroszewski JW. Identification of natural products using HPLC-SPE combined with CapNMR. Anal Chem 2007; 79: 727-735

57 Bringmann G, Messer K, Saeb W, Petes EM, Peters $K$. The absolute configuration of (1)-isoshinanolone and in situ LC-CD analysis of its stereoisomers from crude extracts. Phytochemistry 2001; 56: 387-391

58 Hamburger $M$, Hostettmann K. Bioactivity in plants: the link between phytochemistry and medicine. Phytochemistry 1991; 30: 3864-3874

59 Hostettmann K, Marston A, Wolfender JL. Strategy in the search for new biologically active plant constituents. In: Hostettmann K, Marston A, Maillard M, Hamburger M, editors. Phytochemistry of plants used in traditional medicine. Oxford: Oxford Science Publications; 1995: 1745

60 Xing J, Xie C, Lou H. Recent applications of liquid chromatography-mass spectrometry in natural products bioanalysis. J Pharm Biomed Anal 2007: 44: 368-378

61 Marston A, Maillard M, Hostettmann K. The role of TLC in the investigations of medicinal plants of Africa, South America and other tropical regions. GIT Laboratory J 1997; 1: 36-39

62 Poole CF. Thin-layer chromatography: challenges and opportunities. J Chromatogr A 2003; 1000: 963-984

63 Homans AL, Fuchs A. Direct bioautography on thin layer chromatograms as a method for detecting fungitoxic substances. J Chromatogr 1970; 51: 327-329

64 Marston A, Kissling J, Hostettmann K. A rapid TLC bioautographic method for the detection of acetylcholinesterase and butyrylcholinesterase inhibitors in plants. Phytochem Anal 2002; 13: 51-54

65 Koleva II, Niederländer HAG, van Beek TA. An on-line HPLC method for detection of radical scavenging compounds in complex mixtures. Anal Chem 2000; 72: 2323-2328

66 Pukalskas A, van Beek TA, de Waard P. Development of a triple hyphenated HPLC-radical scavenging detection-DAD-SPE-NMR system for the rapid identification of antioxidants in complex plant extracts. J Chromatogr A 2005; 1074: 81-88 